\title{
Anatomic considerations for immediate implant placement in the mandibular molar region: a cross-sectional study using cone-beam computed tomography
}

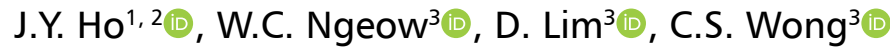 \\ ${ }^{1}$ Department of Restorative Dentistry, Faculty of Dentistry, University of Malaya, Kuala Lumpur, Malaysia \\ ${ }^{2}$ Division of Restorative Dentistry, School of Dentistry, International Medical University, Kuala Lumpur, Malaysia \\ ${ }^{3}$ Department of Oral and Maxillofacial Clinical Sciences, Faculty of Dentistry, University of Malaya, Kuala Lumpur, Malaysia
}

[Received: 4 April 2021; Accepted: 23 May 2021; Early publication date: 14 June 2021]

Background: There is concern regarding immediate implantation in the molar region because of discrepancy between socket size and inserted implant diameter. The purpose of this study was to assess the local anatomy of the posterior mandibular region in relation to immediate implant placement using cone-beam computed tomography (CBCT).

Materials and methods: Using CBCT imaging data, 204 mandibular first molars and 201 mandibular second molars were assessed for the interradicular and alveolar bone dimensions, tooth sizes and proximity to vital structures. The cross-sectional mandibular shape and root configuration of these molars were determined. Results: Distances to the inferior alveolar canal (IAC) from the root apices of the first molar were significantly greater than the second molar. Up to $14.5 \%$ of second molars had less than $10 \mathrm{~mm}$ of vertical bone height between the IAC and furcation bone crest. Interradicular bone width of $<3 \mathrm{~mm}$ was found in $57 \%$ of second molars. All first molars in this study had two to three roots while $16 \%$ of second molars presented with a single root. The prevalent mandible shape at the first and second molars was the parallel and undercut ridges, respectively. Conclusions: The mandibular second molars from samples of a Southeast Asian population presented with greater anatomical difficulties for immediate implant placement which include absent or inadequate interradicular bone thickness, higher incidence of unfavourable mandible shape and increased proximity to vital structures. (Folia Morphol 2022; 81, 3: 732-738)

Key words: cone-beam computed tomography, immediate dental implant loading, mandible, molar

\section{INTRODUCTION}

Dental implant is a popular treatment modality for replacing missing teeth. Immediate implant placement (IIP) protocol has risen in popularity because it shortens treatment time and reduces the number of surgeries required. However, there is concern regarding immediate implantation in the molar region as there is a discrepancy between the socket size and diameter of implant inserted. The primary stability in such cases is achieved by engaging the implant

Address for correspondence: Prof. Dr. W.C. Ngeow, Department of Oral and Maxillofacial Clinical Sciences, Faculty of Dentistry, University of Malaya, 50603, Kuala Lumpur, Malaysia, tel: 603-79674862, fax: 603-79674548, e-mail: ngeowy@um.edu.my

This article is available in open access under Creative Common Attribution-Non-Commercial-No Derivatives 4.0 International (CC BY-NC-ND 4.0) license, allowing to download articles and share them with others as long as they credit the authors and the publisher, but without permission to change them in any way or use them commercially. 
fixture into the inter-radicular septal bone. Factors such as the proximity of the inferior alveolar canal (IAC), socket morphology, the availability of adequate inter-radicular septal bone and the presence of lingual concavities need to be considered prior to IIP at the mandibular molar region [15].

The advent of cone-beam computed tomography $(C B C T)$ has revolutionised craniofacial imaging. CBCT presents clinicians with high resolution images of anatomical structures such as bone topography, periodontal ligament, and root morphology. In addition, the CBCT DICOM data generated can be used to design and fabricate a three-dimensional surgical guide to facilitate implant placement in a prosthetically driven position [11].

Previous studies on the morphology of posterior mandible in relation to IIP had been conducted primarily among Caucasoid populations [1, 2, 5-7, 9, 12]. Information pertaining to the Mongoloid (Southeast Asia) population remains scarce. In addition, not all studies looked into the interradicular bone, which is one of the primary areas of bone available for immediate implant anchorage.

This study aimed to evaluate the morphological features of mandibular first (M1) and second (M2) molars and their surrounding structures in a Mongoloid (Southeast Asian) population within the context of immediate implant placement, using CBCT images.

\section{MATERIALS AND METHODS}

A cross-sectional CBCT study was conducted at the Department of Oral and Maxillofacial Clinical Sciences, Faculty of Dentistry, University of Malaya between May 2020 and October 2020. Ethical approval was received from the Medical Ethics Committee, Faculty of Dentistry with reference number: DF OS2020/081(L). All patients whose CBCT data was used in this study had provided written consent agreeing to release their imaging data for research/academic purposes.

Cone-beam computed tomography imaging data of patients who visited the Oral Radiology Unit, Faculty of Dentistry, University of Malaya between 2010 and 2015 was screened. Included were Malaysian patients of different ethnicities, aged between 18 and 60 years old, without mandibular deformities, and presenting with M1 and M2 molars on either/both sides of the lower jaw. Excluded were subjects with history of dentoalveolar trauma or mandibular pathology, mixed dentition, poor quality CBCTs and evidence of surgical intervention to the mandible.
The sample size was calculated with the following formula [4]:

$$
\text { Sample size }=\frac{Z_{1-\alpha / 2}^{2} S D^{2}}{d^{2}}
$$

where $Z_{1-\alpha}=$ standard normal variate; $S D=$ standard deviation of variable measured; $d=$ absolute error or precision.

Based on the standard deviation of 2.61 from a previous study by Chrcanovic et al. [5]:

$$
\text { The sample size }=\frac{1.96^{2} \times 2.61^{2}}{0.5^{2}}=104.7
$$

Primary outcomes were the morphometric measurements of the alveolar and interradicular bone of $\mathrm{M} 1$ and $\mathrm{M} 2$ and their cross-sectional mandible shapes. Secondary outcomes included proximity to the IAC, root configuration and tooth dimensions.

The CBCT scans were captured using the i-CAT Vision system developed by Imaging Sciences International (Pennsylvania, United States). All images were taken according to a standard protocol. The exposure parameter (120 KvP, 3-7 mA, $20 \mathrm{~s}$ ) and the image acquisition at $0.3 \mathrm{~mm}$ voxel size were done by the same radiographer. The images were obtained from scans acquired with $16 \mathrm{~cm}$ (diameter) and $13 \mathrm{~cm}$ (height) dimensions, and the DICOM was reconstructed using proprietary i-CAT image reconstruction software. The following measurements were made: root length, distance between cementoenamel junction and the separation lines of the root cones, distance between separation lines of root cones to root apex (Fig. 1A), bucco-lingual and mesio-distal crown width (Fig. 1E), interradicular septal bone thickness (Fig. 2A), bucco-lingual width of cancellous portion of the alveolar bone (Fig. 2B), distance from the IAC to the crest of the interradicular septum and root apex (Fig. 2C, D). The mandible ridge form at M1 and M2 was classified into convex, parallel or undercut type, based on the description by Chan et al. [3] (Fig. 3A-C). The root configurations of $\mathrm{M} 1$ and $\mathrm{M} 2$ were visualised on cross-sectional slices to detect the presence of $\mathrm{C}$-shaped root, single fused root, or additional roots (Fig. 3D). Data analysis was completed using IBM SPSS Statistics software version 20 .

The radiographic measurements were performed by the principal examiner (H.J.Y.) with 3 years of post-graduation experience. For reliability testing, intraclass correlation coefficient (ICC) was determined according to the single measurement, absolute agreement, 2-way mixed effects model by repeating the 


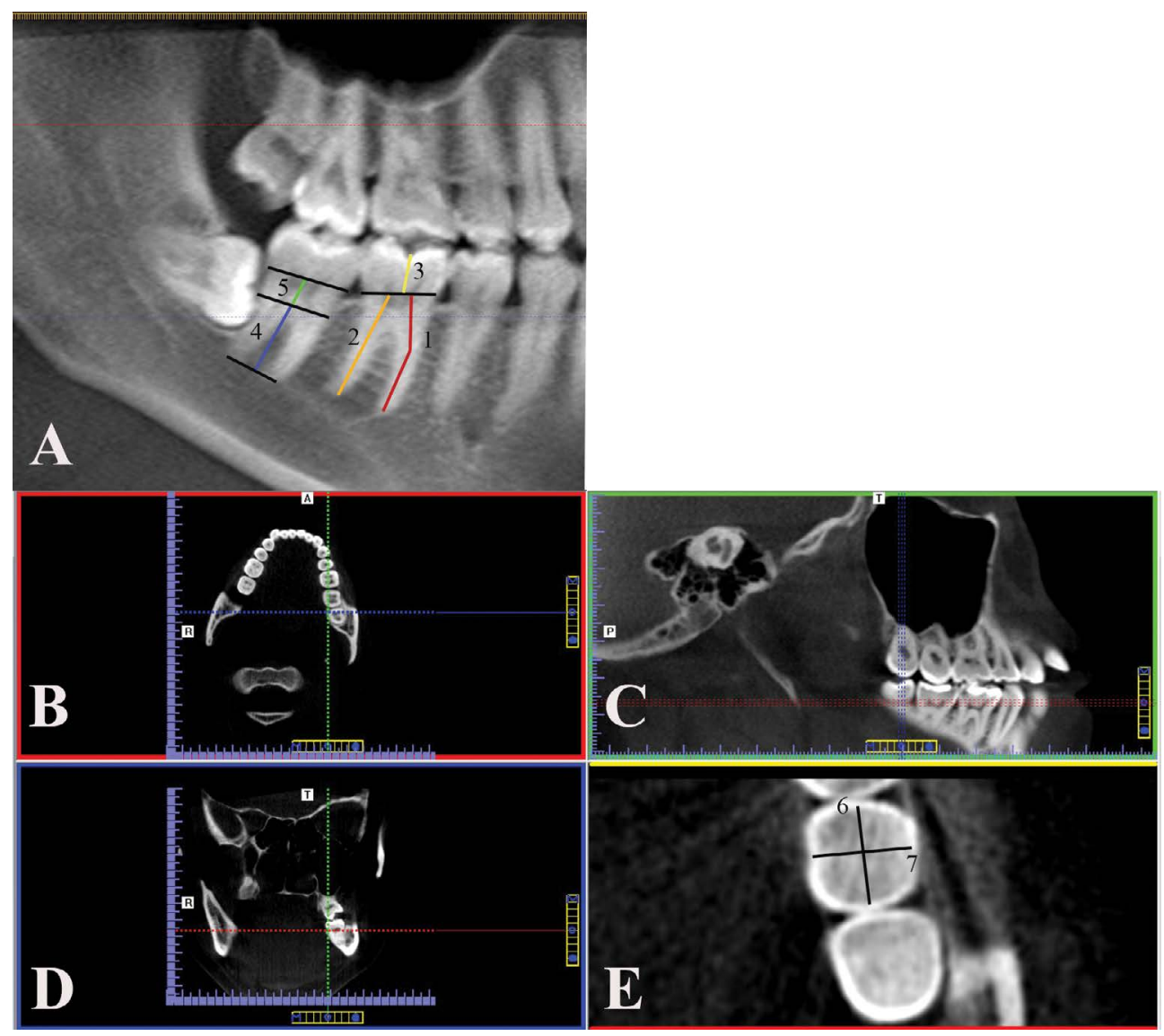

Figure 1. Quantitative measurement of crown and root dimensions; A. Panoramic view; B. Axial view; C. Sagittal view; D. Coronal view; E. Additional cut planes; 1 - mesial root length; 2 - distal root length; 3 - crown height; 4 - root cone; 5 - root trunk; 6 - mesio-distal crown width; 7 - bucco-lingual crown width.
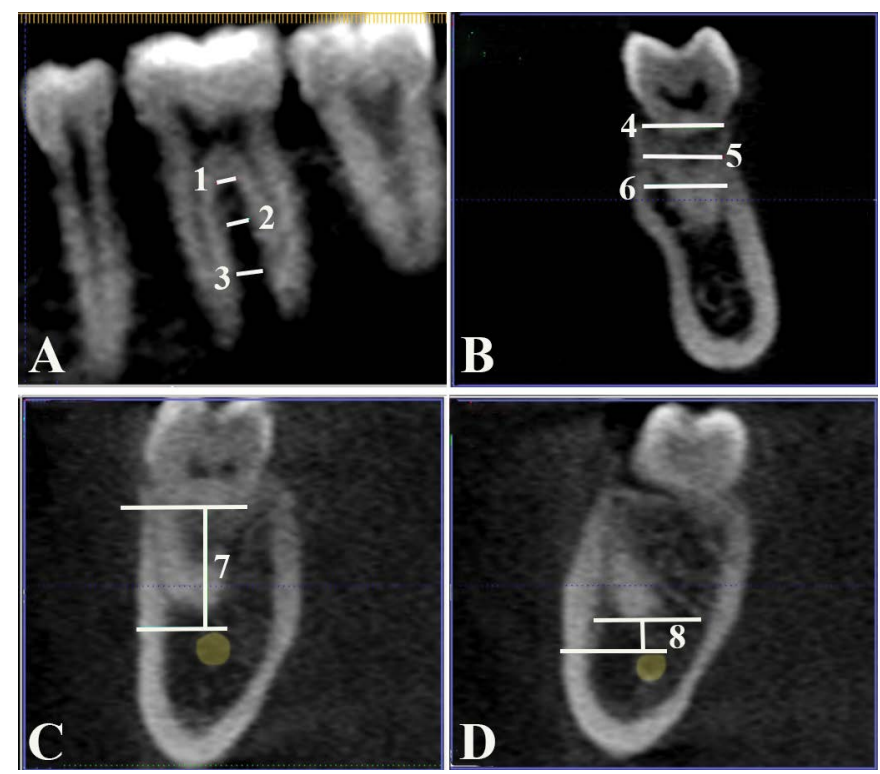

Figure 2. Sagittal and coronal section of the mandible;

A. Mesio-distal width of interradicular bone at three different levels: crest of interradicular septum (1), $3 \mathrm{~mm}$ apical to interradicular septum crest (2), and $6 \mathrm{~mm}$ apical to interradicular septum crest (3); B. Bucco-lingual width of cancellous bone at three different levels: alveolar crest (4), $3 \mathrm{~mm}$ apical to alveolar crest (5) and $6 \mathrm{~mm}$ apical to alveolar crest (6);

C. Distance between interradicular septum crest and inferior alveolar canal (7); D. Distance between root apex and inferior alveolar canal (8). measurements for 30 datasets 2 weeks after the initial measurements. The ICC was 0.985 with a $95 \%$ confidence interval $(\mathrm{Cl})$ of $0.982-0.988$. Therefore, the intra-rater reliability was excellent. An external examiner (W.C.S.) with 5 years of clinical experience was enlisted to determine the inter-rater reliability. 


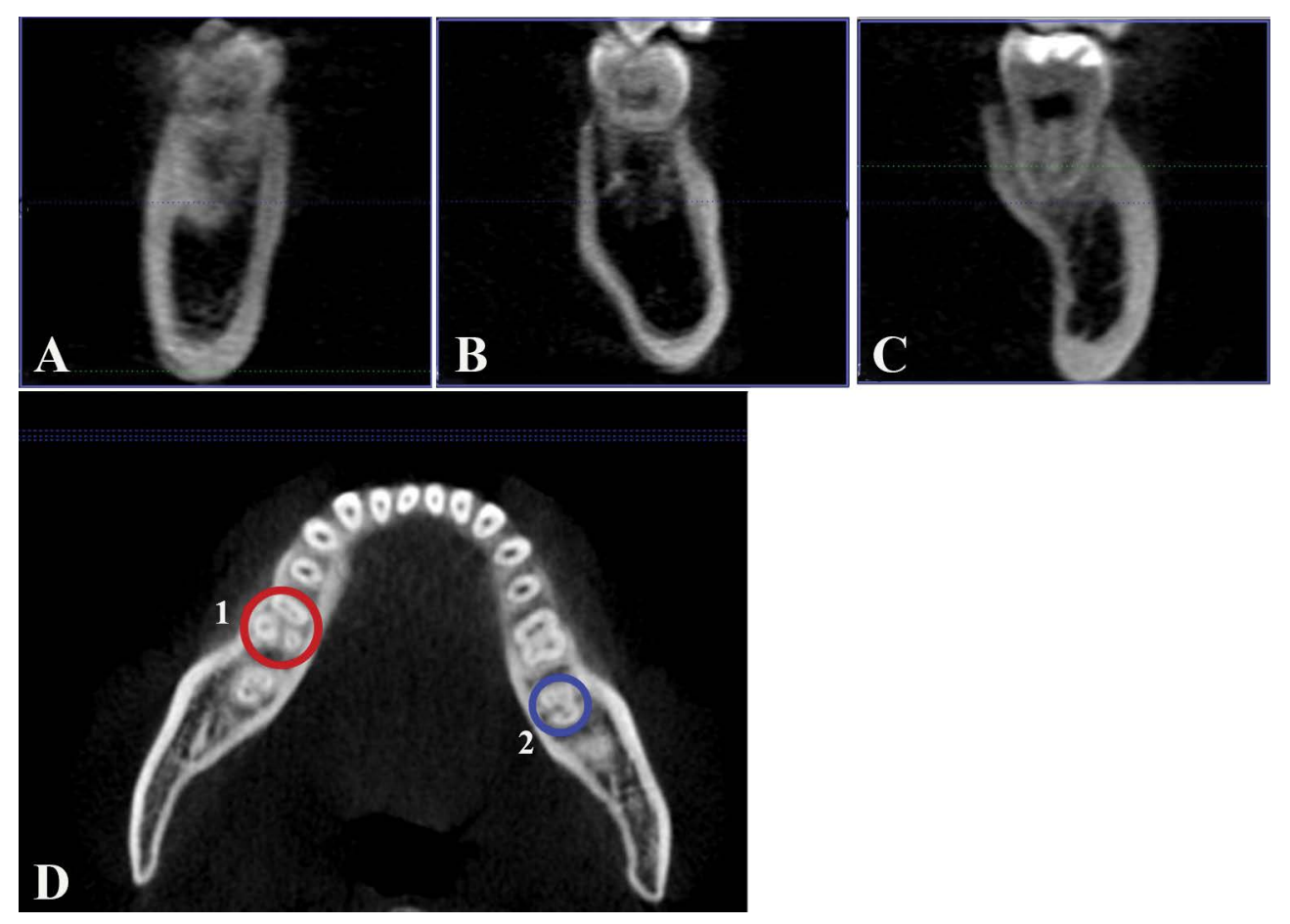

Figure 3. Alveolar ridge classification and anatomical variation of mandibular molar roots; A. Parallel type; B. Convergent type; C. Undercut type; D. 1 - radix entomolaris; $2-\mathrm{C}$-shaped root.

Table 1. Demographic data of study population

\begin{tabular}{llc}
\hline Age [year] (mean \pm SD) & & $36 \pm 11.92$ \\
Gender, n (\%) & Male & $70(63.6 \%)$ \\
& Female & $40(36.4 \%)$ \\
Race, n (\%) & Malay & $46(41.8 \%)$ \\
& Chinese & $37(33.6 \%)$ \\
& Indian & $22(20 \%)$ \\
& Others & $5(4.5 \%)$ \\
\hline
\end{tabular}

SD - standard deviation

Both of them had been calibrated with the senior supervisor in oral and maxillofacial surgery with 14 years of experience in using CBCT (W.C.N.) prior to the commencement of this study. The same ICC model was calculated, resulting in the ICC of 0.94 with a $95 \% \mathrm{Cl}$ of $0.88-0.97$. Therefore, the level of reliability between different examiners was deemed as good to excellent.

Frequency distribution and descriptive statistics for each measurement were calculated. The normality of data was assessed using the Kolmogorov-Smirnov test. Independent samples t-test and Pearson $\chi^{2}$ test were used to compare findings between groups. A p-value of less than 0.05 was considered as statistically significant.

\section{RESULTS}

The study population had a mean age of $35.9 \pm$ \pm 11.9 years. There were more males $(64.5 \%)$ than females (35.5\%) whose CBCT were included. The greatest proportion of the participants was Malay (41.8\%), followed by the Chinese (33.6\%) and Indian (20\%) ethnicity (Table 1$)$.

Two hundred four M1 and 201 M2 were analysed, with no significant difference found between contralateral sides $(p>0.05)$. The crown size of $M 1$ was not significantly different than $M 2$. All M1 had divergent roots while $16 \%$ of $M 2$ were found to have fused roots (Table 2 ). M2 had significantly reduced interradicular bone thickness, greater alveolar bone width and closer proximity to the IAC compared to M1 ( $p<0.001$ ) (Fig. 4). Interradicular bone width $<3 \mathrm{~mm}$ was found in $76.8 \%$ of $\mathrm{M} 2$ and $44.6 \%$ of $\mathrm{M} 1$ (Table 3). Furcation to IAC distance of less than $10 \mathrm{~mm}$ was found in $3 \%$ of $M 1$ and $13.4 \%$ of $M 2$ (Table 3 ).

There was a significant association between tooth type and both ridge form and root configuration $(p<0.001)$. The most common ridge form at the M1 region was the parallel type (70.2\%). The undercut ridge form was found in the majority $(73.1 \%)$ of $\mathrm{M} 2$ (Table 2). The distance to the IAC of female subjects was shorter when measured from the crest of the 
Table 2. Tooth dimensions, mandible shape and root configuration of the first and second mandibular molars

\begin{tabular}{|c|c|c|c|}
\hline & $\begin{array}{c}M 1 \\
\text { (mean } \pm \text { SD) }\end{array}$ & $\begin{array}{c}\text { M2 } \\
\text { (mean } \pm \text { SD) }\end{array}$ & P-value ${ }^{a}$ \\
\hline \multicolumn{4}{|l|}{ Crown size $[\mathrm{mm}]$ : } \\
\hline Height & $5.65 \pm 0.90$ & $5.74 \pm 0.86$ & 0.30 \\
\hline Mesio-distal & $10.45 \pm 0.76$ & $10.22 \pm 0.77$ & $0.002^{*}$ \\
\hline Bucco-lingual & $9.41 \pm 0.86$ & $9.44 \pm 0.76$ & 0.74 \\
\hline \multicolumn{4}{|l|}{ Root length [mm]: } \\
\hline Mesial & $12.83 \pm 1.64$ & $11.61 \pm 1.76$ & $<0.001^{*}$ \\
\hline Distal & $12.31 \pm 1.54$ & $11.04 \pm 1.56$ & $<0.001^{*}$ \\
\hline \multicolumn{4}{|l|}{ Root complex [mm]: } \\
\hline Root trunk & $3.33 \pm 0.55$ & $3.36 \pm 0.51$ & 0.64 \\
\hline \multirow[t]{2}{*}{ Root cone } & $9.83 \pm 1.19$ & $8.83 \pm 1.17$ & $<0.001^{*}$ \\
\hline & $\begin{array}{c}\text { M1 } \\
\text { n (\%) }\end{array}$ & $\begin{array}{c}\text { M2 } \\
\text { n (\%) }\end{array}$ & P-value ${ }^{b}$ \\
\hline \multicolumn{4}{|c|}{ Mandible cross-sectional shape: } \\
\hline Parallel & $138(70.8 \%)$ & $48(25.3 \%)$ & $<0.001^{*}$ \\
\hline Convergent & $9(4.6 \%)$ & $2(1.1 \%)$ & \\
\hline Undercut & $48(24.6 \%)$ & $140(73.7 \%)$ & \\
\hline \multicolumn{4}{|l|}{ Root configuration: } \\
\hline Single conical root & $0(0 \%)$ & $11(5.7 \%)$ & $<0.001^{*}$ \\
\hline Double roots & $174(89.7 \%)$ & $162(83.5 \%)$ & \\
\hline Three roots & $20(10.3 \%)$ & $1(0.5 \%)$ & \\
\hline C-shaped root & $0(0 \%)$ & $29(10.3 \%)$ & \\
\hline
\end{tabular}

${ }^{*}$ Denote statistical significance $(p<0.05)$; alndependent samples $\mathrm{t}$-test; ${ }^{\mathrm{b}}$ Pearson $\chi^{2}$ test; M1 - mandibular 1 15t molar; M2 - mandibular 2 $2^{\text {nd }}$ molar; SD — standard deviation

Table 3. Proportion of teeth with compromised recipient site for immediate implants

\begin{tabular}{lcccc}
\hline Tooth & $\begin{array}{c}\text { IRB } \\
<\mathbf{3 ~} \mathbf{~ m m}\end{array}$ & $\begin{array}{c}\text { F-IAC } \\
<\mathbf{1 0} \mathbf{~ m m}\end{array}$ & $\begin{array}{c}\text { M-IAC } \\
<\mathbf{2} \mathbf{~ m m}\end{array}$ & $\begin{array}{c}\text { D-IAC } \\
<\mathbf{2} \mathbf{~ m m}\end{array}$ \\
\hline M1 & $86 / 193$ & $6 / 204$ & $14 / 204$ & $18 / 204$ \\
& $(44.6 \%)$ & $(3 \%)$ & $(6.9 \%)$ & $(8.8 \%)$ \\
M2 & $116 / 151$ & $27 / 201$ & $40 / 201$ & $67 / 191$ \\
& $(76.8 \%)$ & $(13.4 \%)$ & $(20 \%)$ & $(35 \%)$ \\
\hline
\end{tabular}

M1 - mandibular $1^{\text {st }}$ molar; M2 - mandibular $2^{\text {nd }}$ molar; IRB — interradicular bone width; F-IAC - bone height between furcation crest and inferior alveolar canal; $\mathrm{M}-\mathrm{IAC}$ - bone height between mesial root apex and inferior alveolar canal; $\mathrm{D}-\mathrm{IAC}$ - bone height between distal root apex and inferior alveolar canal

interradicular septum and mesial root apex $(p<0.05)$. On the other hand, males possessed greater root cone height of M1 and M2 than females $(p<0.05)$.

\section{DISCUSSION}

In immediate molar implantation, the socket size is large when compared to the diameter of standard implants. When inter-radicular septal bone is
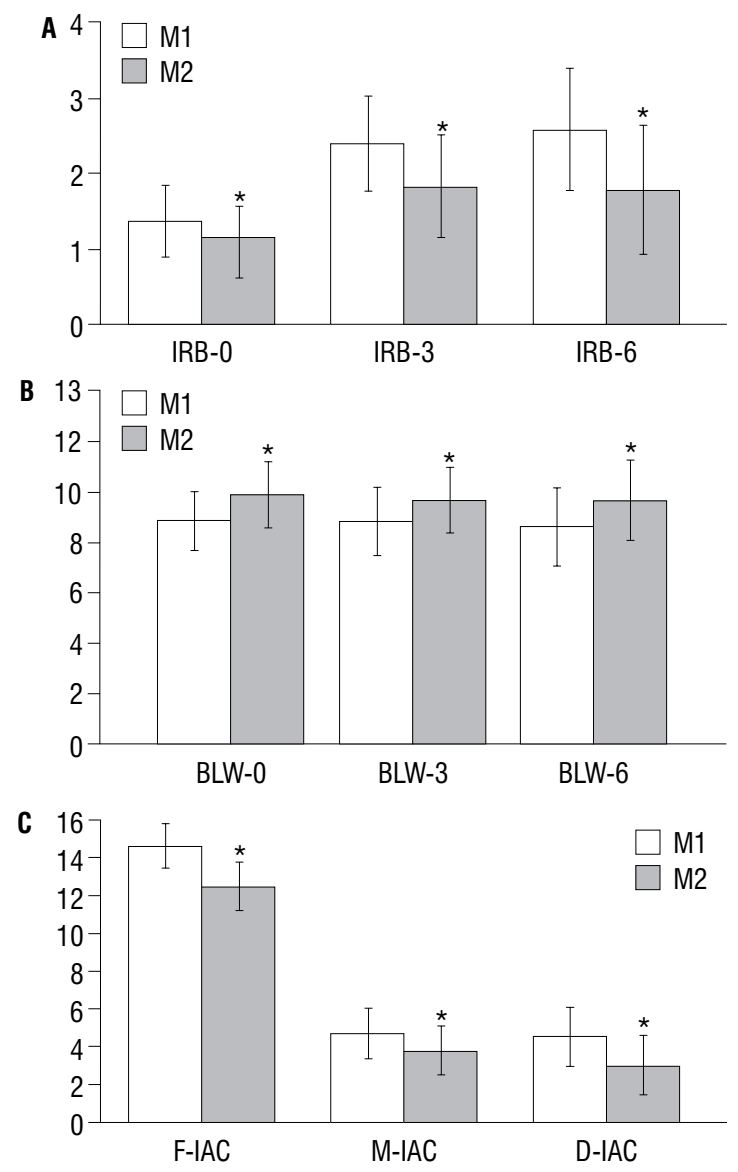

Figure 4. Bar graph demonstrating interradicular bone width (A), alveolar bone width (B) and proximity to inferior alveolar canal for mandibular first (M1) and mandibular second (M2) (C); IRB-0 interradicular bone width at furcation crest; IRB-3 - interradicular bone width $3 \mathrm{~mm}$ apical to furcation crest; IRB-6 - interradicular bone width $6 \mathrm{~mm}$ apical to furcation crest; BLW-0 - bucco-lingual alveolar bone width at alveolar crest; BLW-3 - bucco-lingual alveolar bone width $3 \mathrm{~mm}$ apical to alveolar crest; BLW-6 bucco-lingual alveolar bone width $6 \mathrm{~mm}$ apical to alveolar crest; F-IAC - bone height between furcation crest and inferior alveolar canal; M-IAC - bone height between mesial root apex and inferior alveolar canal; D-IAC — bone height between distal root apex and inferior alveolar canal; *indicates statistically significant difference between $\mathrm{M} 1$ and $\mathrm{M} 2(\mathrm{p}<0.05)$.

inadequate to provide primary stability, clinicians are advised to insert implant fixtures into the bone beyond the inter-dental base to achieve primary stability [15].

Dimensional changes of the external socket walls were reported to be more pronounced at the buccal aspect following IIP of molars [10]. Bucco-lingually, we observed a cancellous bone width of $>8.5 \mathrm{~mm}$ at $\mathrm{M} 1$ and $>9.5 \mathrm{~mm}$ at M2, so hypothetically, even when resorption is factored in, there shall be adequate bone to receive a wide diameter $(\geq 4.5 \mathrm{~mm})$ implant with little risk of thread exposure bucco-lin- 
gually [13]. Mesiodistally, both M1 and M2 showed incremental width of the inter-radicular septal bone apically, due to the mesio-distal convergence of their roots. The average distance from the crest of the interradicular septum to the IAC was greater than $12 \mathrm{~mm}$ for both M1 and M2. A distance of less than $10 \mathrm{~mm}$ was found in $2.7 \%$ of M1 sites and $14.5 \%$ of $M 2$ sites. Taken together these findings suggested that it is safe to insert a $10 \mathrm{~mm}$-length implant into the inter-radicular septal bone of $\mathrm{M} 1$ without risking protrusion into the IAC. Combined with the finding that parallel mandible shape was predominant in about $70 \%$ of $\mathrm{M} 1$, the risk of immediate implant perforation into the sublingual fossa is lesser in M1 than M2.

As the prevalent mandible shape at $\mathrm{M} 2$ was the undercut type (73.1\%), immediate implantation at this site is accompanied by a higher risk of lingual plate perforation. Moreover, $16 \%$ of M2 had a single root; therefore the immediate implant cannot engage the inter-radicular septal bone as it is non-existent. Instead, it shall be inserted into the apical bone. Vertically, most literatures recommended that implant should be placed at least $>3 \mathrm{~mm}$ apical to the extraction site [14]. Extra precaution is warranted, because even for two-rooted M2, the mesial and distal roots to IAC distances for M2 were on average, $3.78 \pm 2.31 \mathrm{~mm}$ and $3.03 \pm 2.24 \mathrm{~mm}$, respectively. These reduced distances as compared to M1 may not permit immediate implant placement into the socket of $\mathrm{M} 2$ without risking damage to the inferior alveolar neurovascular bundle.

While the above information suggested that vertically it is safe to place a $10 \mathrm{~mm}$ long implant into the inter-radicular septal bone, its mesio-distal width is just sufficient to receive a standard diameter implant. Smith and Tarnow [15] proposed a classification system for molar extraction sites for immediate implant placement. Type A socket is the situation when an implant is completely fixed within the septal bone, without gaps between the implant and the socket walls. In type B socket, the implant has adequate but incomplete septal bone, resulting in gaps following implant insertion. Lastly, type $C$ socket has insufficient septal bone, resulting in the need to engage the implant at the periphery of extraction sockets [15]. The current findings suggested that the majority of extraction sockets belonged to type $\mathrm{B}$ in $\mathrm{M} 1$ and $\mathrm{M} 2$, with type $\mathrm{C}$ observed in $16 \%$ of $\mathrm{M} 2$. In type C sockets, the primary stability will be provided by buccal, lingual and apical trabecular bone.

The geometry and anatomy of the mandible are crucial aspects that need to be considered carefully prior to immediate implantation. Previous studies observed that the undercut shaped ridge was the most common mandibular geometry at the posterior region $[5,9]$. According to a virtual IIP simulation study, lingual bone plate perforation was more prevalent in $\mathrm{U}$-shaped ridges, and more frequently affected the M2 sites $[8,16]$. Similar findings regarding the anatomical limitations of $\mathrm{M} 2$ in relation to the IAC and sublingual fossa were observed in this study, which confirmed the findings of several studies $[5-7,9]$. In contrast, the present study found that M2 demonstrated greater bucco-lingual width than M1. This broad alveolar crest observed easily allows for delayed implant treatment protocol. All facts considered, more M2 sockets observed in this study were not ideal for immediate implantation, as compared to $\mathrm{M} 1$.

Regarding gender differences in the parameters measured, our findings suggested that the distance of the IAC to the interradicular bony septum crest and mesial root apex was significantly lesser among female subjects. Therefore, female patients will face a higher risk of inferior alveolar neurovascular bundle injury when the apical bone was used to achieve primary stability.

\section{Limitations of the study}

The limitation of this study is that non-probability sampling method was used which might introduce selection bias. Therefore, inferences drawn from the data for the entire Malaysian population should be interpreted with caution. Moreover, the exact implications of these anatomical features during immediate implantation could be better appreciated with virtual implant simulation.

\section{CONCLUSIONS}

This study showed that the inter-radicular bone of two-rooted M1 and M2, and the periphery of M2 sockets with fused roots are possible sites for immediate implant placement. However, M2 sockets may be less ideal for immediate implantation on the account of their variable anatomy.

\section{Conflict of interest: None declared}

\section{REFERENCES}

1. Agostinelli C, Agostinelli A, Berardini M, et al. Radiological evaluation of the dimensions of lower molar alveoli. Implant Dent. 2018; 27(3): 271-275, doi: 10.1097/ ID.0000000000000757, indexed in Pubmed: 29652756. 
2. Braut $\mathrm{V}$, Bornstein $\mathrm{MM}$, Lauber $\mathrm{R}$, et al. Bone dimensions in the posterior mandible: a retrospective radiographic study using cone beam computed tomography. Part 1 - analysis of dentate sites. Int J Periodontics Restorative Dent. 2012; 32(2): 175-184, indexed in Pubmed: 22292147.

3. Chan HL, Brooks SL, Fu JH, et al. Cross-sectional analysis of the mandibular lingual concavity using cone beam computed tomography. Clin Oral Implants Res. 2011; 22(2): 201-206, doi: 10.1111/j.1600-0501.2010.02018.x, indexed in Pubmed: 21044167.

4. Charan J, Biswas T. How to calculate sample size for different study designs in medical research? Indian J Psychol Med. 2013; 35(2): 121-126, doi: 10.4103/02537176.116232, indexed in Pubmed: 24049221.

5. Chrcanovic BR, de Carvalho Machado V, Gjelvold B. Immediate implant placement in the posterior mandible: A cone beam computed tomography study. Quintessence Int. 2016; 47(6): 505-514, doi: 10.3290/j.qi.a36008, indexed in Pubmed: 27092357.

6. Froum S, Casanova L, Byrne $S$, et al. Risk assessment before extraction for immediate implant placement in the posterior mandible: a computerized tomographic scan study. J Periodontol. 2011; 82(3): 395-402, doi: 10.1902/ jop.2010.100360, indexed in Pubmed: 20809864.

7. Haj Yahya B, Chaushu G, Hamzani Y. Computed tomography for the assessment of the potential risk after implant placement in fresh extraction sites in the posterior mandible. J Oral Implantol. 2021; 47(1): 2-8, doi: 10.1563/ aaid-joi-D-18-00227, indexed in Pubmed: 32662837.

8. Huang RY, Cochran DL, Cheng WC, et al. Risk of lingual plate perforation for virtual immediate implant placement in the posterior mandible: A computer simulation study. J Am Dent Assoc. 2015; 146(10): 735-742, doi: 10.1016/j. adaj.2015.04.027, indexed in Pubmed: 26409983.

9. Lin MH, Mau LP, Cochran DL, et al. Risk assessment of inferior alveolar nerve injury for immediate implant placement in the posterior mandible: a virtual implant placement study. J Dent. 2014; 42(3): 263-270, doi: 10.1016/j. jdent.2013.12.014, indexed in Pubmed: 24394585.

10. Matarasso S, Salvi GE, lorio Siciliano V, et al. Dimensional ridge alterations following immediate implant placement in molar extraction sites: a six-month prospective cohort study with surgical re-entry. Clin Oral Implants Res. 2009; 20(10): 1092-1098, doi: 10.1111/j.16000501.2009.01803.x, indexed in Pubmed: 19719737.

11. Nagarajan A, Perumalsamy R, Thyagarajan R, et al. Diagnostic imaging for dental implant therapy. J Clin Imaging Sci. 2014; 4(Suppl 2): 4, doi: 10.4103/2156-7514.143440, indexed in Pubmed: 25379354.

12. Padhye NM, Shirsekar VU, Bhatavadekar NB. Three-dimensional alveolar bone assessment of mandibular first molars with implications for immediate implant placement. Int J Periodontics Restorative Dent. 2020; 40(4): e163-e167, doi: 10.11607/prd.4614, indexed in Pubmed: 32559042.

13. Renouard F, Nisand D. Impact of implant length and diameter on survival rates. Clin Oral Implants Res. 2006; 17(Suppl 2): 35-51, doi: 10.1111/j.1600-0501.2006. 01349.x, indexed in Pubmed: 16968380.

14. Schwartz-Arad D, Chaushu G. The ways and wherefores of immediate placement of implants into fresh extraction sites: a literature review. J Periodontol. 1997; 68(10): 915-923, doi: 10.1902/jop.1997.68.10.915, indexed in Pubmed: 9358358.

15. Smith RB, Tarnow DP. Classification of molar extraction sites for immediate dental implant placement: technical note. Int J Oral Maxillofac Implants. 2013; 28(3): 911-916, doi: 10.11607/jomi.2627, indexed in Pubmed: 23748327.

16. Wang TY, Kuo PJ, Fu E, et al. Risks of angled implant placement on posterior mandible buccal/lingual plated perforation: A virtual immediate implant placement study using CBCT. J Dent Sci. 2019; 14(3): 234-240, doi: 10.1016/j. jds.2019.03.005, indexed in Pubmed: 31528250. 